\section{Estudo comparativo das indicações de cesariana entre um hospital público- universitário e um hospital privado}

\section{Comparative study of cesarean section indications between a public university hospital and a private hospital}

Renato Humberto Fabri 1

Helder de Souza Lima e Silva 2

Rodrigo Vaz de Lima 2

Eddie Fernando Candido Murta 3

1 Departamento de Medicina Social. Faculdade de Medicina do Triângulo Mineiro. Rua Getúlio Guaritá, s. n. Abadia. Uberaba, Minas Gerais, Brasil. CEP 38.025-440. E-mail: renatofabri@mednet.com.br

2 Faculdade de Medicina do Triângulo Mineiro

3 Disciplina de Ginecologia e Obstetrícia da Faculdade de Medicina do Triângulo Mineiro

\section{Abstract}

Objectives: to analyse the incidence and the indications of cesarean section performed in the University Hospital of the "Triângulo Mineiro Faculty of Medicine " and a private hospital in Uberaba, Minas Gerais, Brazil.

Methods: a cross sectional study was carried out by using the data source of the medical files from both hospitals consisting of patient age, place of origin, marital status, education level, parity and cesarean sections indications.

Results: the cesarean sections rate was $24,3 \%$ (325 of 1.441 births) at the university hospital against 89,2\% (100 of 112 births) of the private hospital. The most frequent indications in the University Hospital were previous cesarean sections $(26,7 \%)$, dystocia $(22,4 \%)$ and acute fetal distress (18,2\%). In the private hospital, indications were previous cesarean section (36\%) and dystocia (36\%). The private hospital's patients had higher education levels.

Conclusions: the higher rate of cesarean sections observed in the private hospital resulted from previous cesarean section, dystocia and higher education levels.

Key words Cesarean section, Socioeconomic factors, Women's health

\section{Resumo}

Objetivos: analisar a incidência e as indicações de cesariana realizadas no Hospital Escola da Falculdade de Medicina do Triângulo Mineiro e um hospital privado, ambos localizados em Uberaba, Minas Gerais, Brasil.

Métodos: trata-se de estudo transversal desenvolvido a partir da coleta nos prontuários dos hospitais, consistindo da idade, procedência, situação conjugal, escolaridade, paridade e indicações de cesariana.

Resultados: a incidência de cesariana foi de 24,3\% no Hospital Escola contra 89,2\% no hospital privado. As indicações mais freqüentes de cesariana no Hospital Escola foram a cesárea iterativa (26,7\%), distócia $(22,4 \%)$ e o sofrimento fetal agudo $(18,2 \%)$, e no hospital privado foram cesárea iterativa $(36 \%) e$ distócia (36\%). As pacientes do hospital privado tinham maior escolaridade

Conclusões: os dados sugerem que o aumento de cesarianas no hospital privado foi decorrente de iteratividade, distócia e a escolaridade mais elevada das pacientes.

Palavras-chave Cesárea, Fatores socioconômicos, Saúde da mulher 


\section{Introdução}

Nas últimas décadas tem ocorrido, em todo o mundo, uma crescente incidência de operações cesarianas. $\mathrm{Na}$ Dinamarca a incidência elevou-se de 4,1\% em 1976 para 13\% em 19891 e nos Estados Unidos o índice passou de 5,5\% em 1970 para 24,4\% em 1987.2

Miranda et al, 3 em trabalho realizado sobre indicação de cesariana, verificou que o Brasil tem lugar de destaque nesse cenário e apresenta uma das maiores taxas do mundo. O elevado número de cesarianas é hoje um problema de saúde pública e essas taxas têm proporcionado uma maior morbimortalidade materna. ${ }^{4}$ Conforme citado por Souto et al., 5 em estudos realizados nos hospitais do Instituto Nacional de Assistência Médica e Previdência Social (INAMPS), atual Instituto Nacional do Seguro Social (INSS), a variação foi de $14,6 \%$ para $31 \%$ entre 1970 a 1980. No Hospital das Clínicas de Porto Alegre, Rio Grande do Sul, a incidência passou de 26,6\%, em 1984, para 33,3\% em 1985.5 Em Belo Horizonte, no Hospital das Clínicas da Universidade Federal de Minas Gerais, o índice aumentou de $11,95 \%$ em 1970 para 30,4\% em 1987.6 Betiol$^{7}$ observou que 30,4\% dos partos realizados em Ribeirão Preto, São Paulo, em 1978 e 1979 foram cirúrgicos. Fabri e Gomes, 8 em 1993, estudando tipos de parto em Uberaba, Minas Gerais, encontraram uma taxa de cesarianas de $53,2 \%$, sendo considerada elevada quando comparada aos levantamentos bibliográficos nacionais e internacionais.

Entre os vários motivos desse aumento, os mais citados são iteratividade, sofrimento fetal agudo, apresentação pélvica e distócias.3,5,9-13 No Brasil, além destas causas, associa-se a inadequada assistência médica, a precária educação das gestantes; as causas de natureza econômica entre outras, têm determinado o aumento da incidência de cesarianas.

Esse aumento é progressivo e exagerado, ocorrendo principalmente nas camadas socioeconômicas privilegiadas, que frequentam clínicas privadas e/ou têm planos de saúde, enquanto que entre as classes menos favorecidas da população esses índices são menores e ocorrem principalmente nos hospitais públicos ou hospitais escola. 11

A presente investigação se propõem a analisar algumas variáveis ligadas à indicação de cesariana e sua incidência no Hospital Escola da Faculdade de Medicina do Triângulo Mineiro, em Uberaba e em um hospital privado na mesma cidade, comparando os dados entre os dois hospitais.

\section{Métodos}

O presente estudo é do tipo transversal, sendo desenvolvido com a coleta de dados referentes a todas as mulheres que deram à luz por parto cesariana no Hospital Escola (HE) da Faculdade de Medicina do Triângulo Mineiro (FMTM) e em um hospital privado (HA), ambos em Uberaba, no período de janeiro a dezembro de 1996. Foram revistos 1.441 prontuários no HE e 100 no hospital privado.

A pesquisa se realizou a partir do preenchimento de questionários que foram especialmente preparados e constavam de itens relativos à procedência das pacientes (zona urbana ou rural), idade (divididas por faixa etária <20, 20-29, 30-39, 40-50 anos), escolaridade (analfabeto, primeiro grau, segundo grau, nível superior), estado civil (solteira, casada, divorciada, e ignorado), paridade e indicação de cesariana.

Com autorização prévia dos diretores clínicos, os dados foram coletados nos arquivos das instituições envolvidas. Numa fase inicial fez-se o preenchimento de alguns questionários com o objetivo de criar condições de padronização das informações, assim como identificação das dificuldades que pudessem ocorrer no desenvolvimento da investigação.

Sistematicamente, a cada vinte dias o grupo de estudos se reunia para discussão e solução dos problemas referentes ao preenchimento do questionário. Respondido esse questionário, era o mesmo revisado e os dados então codificados, sendo utilizado um manual previamente elaborado. Só então, os dados foram analisados e posteriormente discutidos.

\section{Resultados}

Os dados mostraram que o HE teve a menor proporção de cesarianas $(24,4 \%)$ enquanto que o HA atingiu um índice de 89,3\% (Tabela 1).

Quanto à procedência das pacientes, a maioria dos partos $(86,7 \%)$ foram da zona urbana de Uberaba, e $12 \%$ de outras localidades, sendo $10,5 \%$ da zona urbana destas (Tabela 2).

A distribuição dos partos segundo a idade gestacional mostrou que a grande maioria ocorreu no $\mathrm{HE}$, valor três vezes superior àquele encontrado no HA. Observou-se que aproximadamente $50 \%$ dos partos estão entre aquelas com 20 a 30 anos de idade, tanto no HE quanto no HA (Tabela 3 ).

Pode-se observar também, que a distribuição etária não é homogênea entre os hospitais, e o HE tem uma clientela mais jovem, onde $27 \%$ das parturientes são adolescentes. 
Distribuição das puérperas estudadas segundo o tipo de parto. Uberaba, Minas Gerais, Brasil, 1996.

\begin{tabular}{lrrrr}
\hline & \multicolumn{2}{c}{ HE } & & HA \\
\cline { 2 - 5 } Tipos de parto & \multicolumn{1}{c}{$\mathrm{n}$} & $\%$ & $\mathrm{n}$ & $\%$ \\
\hline Normal & 1.089 & 75,6 & 12 & 10,7 \\
Cesariana & 352 & 24,4 & 100 & 112 \\
Total & 1.441 & 100,0 & 100,0 \\
\hline
\end{tabular}

$\mathrm{HE}=$ Hospital Escola, $\mathrm{HA}=$ Hospital Privado

Tabela 2

Distribuição das puérperas que deram a luz por parto cesariana no Hospital Escola da Faculdade de Medicina do Triângulo Mineiro. Uberaba, Minas Gerais, Brasil, 1996.

\begin{tabular}{|c|c|c|c|c|c|}
\hline \multirow{2}{*}{ Localidade } & & \multicolumn{2}{|c|}{ HA } & \multicolumn{2}{|c|}{$\mathrm{HE}$} \\
\hline & & $\mathrm{n}$ & $\%$ & $\mathrm{n}$ & $\%$ \\
\hline Uberaba & (Zona urbana) & 305 & 86,7 & 84 & 84,0 \\
\hline Uberaba & (Zona rural) & 5 & 1,4 & 0 & 0,0 \\
\hline Outra cidade & (Zona urbana) & 37 & 10,5 & 16 & 16,0 \\
\hline Outra cidade & (Zona rural) & 5 & 1,4 & 0 & 0,0 \\
\hline Total & & 352 & 100,0 & 100 & 100,0 \\
\hline
\end{tabular}

HE = Hospital Escola, HA = Hospital Privado

Tabela 3

Distribuição das puérperas submetidas à cesariana segundo a idade e hospital onde ocorreu o parto. Uberaba, Minas Gerais, Brasil,1996.

\begin{tabular}{|c|c|c|c|c|c|c|c|c|c|}
\hline \multirow[b]{3}{*}{ Hospitais } & \multicolumn{8}{|c|}{ Idade } & \multirow[b]{3}{*}{ Total } \\
\hline & \multicolumn{2}{|c|}{$<20$} & \multicolumn{2}{|c|}{20 I- 30} & \multicolumn{2}{|c|}{$30 \mathrm{l}-40$} & \multicolumn{2}{|c|}{40 I- 50} & \\
\hline & $n$ & $\%$ & $n$ & $\%$ & $n$ & $\%$ & $n$ & $\%$ & \\
\hline $\mathrm{HE}$ & 95 & 27,0 & 174 & 49,4 & 77 & 21,9 & 6 & 1,7 & 352 \\
\hline HA & 8 & 8,0 & 51 & 51,0 & 40 & 40,0 & 1 & 1,0 & 100 \\
\hline
\end{tabular}

$\mathrm{HE}=$ Hospital Escola, HA = Hospital Privado 
A Tabela 4 mostra que a situação conjugal solteira predominou entre as pacientes do Hospital Escola, em relação ao hospital privado em todos os grupos etários, ocorrendo o inverso para aquelas com a situação conjugal casada.

Quanto à escolaridade verificou-se que o analfabetismo era pequeno. No HE, entre as mulheres que cursaram até primeiro grau, predominaram as adolescentes, com 79,0\%, diminuindo entre os grupos etários de 20 a 30,30 a 40 e 40 a 50 anos com $73,5 \%, 68,9 \%$ e $66,7 \%$, respectivamente. Para aquelas do HA, os valores foram significativamente diferentes, ocorrendo o inverso para os níveis superiores de escolaridade (Tabela 5).
A distribuição do número de gestações nos grupos etários mostrou que 5\% das informações ficaram prejudicadas (Tabela 6). A ocorrência de três gestações foi mais freqüente nos grupos com até 30 anos de idade, diminuindo o número de gestações nos grupos etários subsequentes (Tabela 6).

Em relação à indicação do tipo de parto, a Tabela 7 mostra que a cesárea iterativa, distócias e o sofrimento fetal predominaram na indicação de cesariana no HE com 26,7\%, 22,4\% e 18,2\% respectivamente, enquanto que no HA as duas primeiras indicações foram superiores ao hospital escola (36\%), porém o sofrimento fetal foi bem inferior (2\%).

Distribuição das puérperas estudadas no Hospital Escola da Faculdade de Medicina do Triângulo Mineiro e no hospital particular, segundo a situação conjugal e idade. Uberaba, Minas Gerais, Brasil (1996).

\begin{tabular}{|c|c|c|c|c|c|c|c|c|c|c|c|c|c|c|c|c|c|c|c|c|}
\hline \multirow{4}{*}{$\begin{array}{l}\text { Estado } \\
\text { Civil }\end{array}$} & \multicolumn{20}{|c|}{ Idade } \\
\hline & \multicolumn{4}{|c|}{$<20$} & \multicolumn{4}{|c|}{$201-30$} & \multicolumn{4}{|c|}{30 I- 40} & \multicolumn{4}{|c|}{40 I- 50} & \multicolumn{4}{|c|}{ Total } \\
\hline & \multicolumn{2}{|c|}{$\mathrm{HE}$} & \multicolumn{2}{|c|}{$\mathrm{HA}$} & \multicolumn{2}{|c|}{$\mathrm{HE}$} & \multicolumn{2}{|c|}{$\mathrm{HA}$} & \multicolumn{2}{|c|}{$\mathrm{HE}$} & \multicolumn{2}{|c|}{$\mathrm{HA}$} & \multicolumn{2}{|c|}{$\mathrm{HE}$} & \multicolumn{2}{|c|}{$\mathrm{HA}$} & \multicolumn{2}{|c|}{$\mathrm{HE}$} & \multicolumn{2}{|c|}{$\mathrm{HA}$} \\
\hline & $n$ & $\%$ & $\mathrm{n}$ & $\%$ & $n$ & $\%$ & $n$ & $\%$ & $\mathrm{n}$ & $\%$ & $\mathrm{n}$ & $\%$ & $\mathrm{n}$ & $\%$ & $n$ & $\%$ & $\mathrm{n}$ & $\%$ & $\mathrm{n}$ & $\%$ \\
\hline Solteira & 59 & 62,2 & 4 & 50,0 & 72 & 41,4 & 5 & 9,8 & 32 & 41,5 & 2 & 5,0 & 3 & 50,0 & 0 & 0 & 16 & 47,1 & 11 & 11,0 \\
\hline Casada & 36 & 37,8 & 3 & 37,5 & 10 & 58,6 & 45 & 88,2 & 44 & 57,2 & 37 & 92,5 & 3 & 50,0 & 1 & 100,0 & 6 & 52,6 & 86 & 86,0 \\
\hline Divorciada & 0 & 0,0 & 0 & 0,0 & 2 & 0,0 & 1 & 2,0 & 1 & 1,3 & 1 & 2,5 & 0 & 0,0 & 0 & 0,0 & 18 & 0,3 & 2 & 2,0 \\
\hline Ignorado & 0 & 0,0 & 1 & 12,5 & 0 & 0,0 & 0 & 0,0 & 0 & 0,0 & 0 & 0,0 & 0 & 0,0 & 0 & 0,0 & 5 & 0,0 & 1 & 1,0 \\
\hline Total & 95 & 100,0 & 8 & 100,0 & 0 & 100,0 & 51 & 100,0 & 77 & 100,0 & 40 & 100,0 & 6 & 100,0 & 1 & 100,0 & 1 & 100,0 & 100 & 100 \\
\hline
\end{tabular}

$\mathrm{HE}=$ Hospital Escola, HA = Hospital Privado 
Distribuição das puérperas estudadas no Hospital Escola da Faculdade de Medicina do Triângulo Mineiro, e no hospital particular segundo o nível de escolaridade e idade. Uberaba, Minas Gerais, Brasil, 1996.

\begin{tabular}{|c|c|c|c|c|c|c|c|c|c|c|c|c|c|c|c|c|c|c|c|c|}
\hline \multicolumn{21}{|c|}{ Idade } \\
\hline & \multicolumn{4}{|c|}{$<20$} & \multicolumn{4}{|c|}{$201-30$} & \multicolumn{4}{|c|}{$30 \mathrm{I}-40$} & \multicolumn{4}{|c|}{40 I- 50} & \multicolumn{4}{|c|}{ Total } \\
\hline & \multicolumn{2}{|c|}{$\mathrm{HE}$} & \multicolumn{2}{|c|}{ HA } & \multicolumn{2}{|c|}{$\mathrm{HE}$} & \multicolumn{2}{|c|}{ HA } & \multicolumn{2}{|c|}{$\mathrm{HE}$} & \multicolumn{2}{|c|}{ HA } & \multicolumn{2}{|c|}{$\mathrm{HE}$} & \multicolumn{2}{|c|}{ HA } & \multicolumn{2}{|c|}{$\mathrm{HE}$} & \multicolumn{2}{|c|}{ HA } \\
\hline & $n$ & $\%$ & $\mathrm{n}$ & $\%$ & $\mathrm{n}$ & $\%$ & $\mathrm{n}$ & $\%$ & $\mathrm{n}$ & $\%$ & $n$ & $\%$ & $\mathrm{n}$ & $\%$ & $\mathrm{n}$ & $\%$ & $\mathrm{n}$ & $\%$ & $n$ & $\%$ \\
\hline Analfabeto & 1 & 1,0 & 0 & 0,0 & 6 & 3,5 & 0 & 0,0 & 2 & 2,6 & 0 & 0,0 & 2 & 33,3 & 0 & 0,0 & 11 & 3,1 & 0 & 0,0 \\
\hline $1^{\circ}$ grau inc. & 69 & 72,7 & 3 & 37,5 & 109 & 62,6 & 1 & 2,0 & 50 & 65,0 & 1 & 2,2 & 4 & 66,7 & 0 & 0,0 & 23 & 66,0 & 5 & 5,0 \\
\hline $1^{\circ}$ grau comp. & 6 & 6,3 & 0 & 0,0 & 19 & 10,9 & 3 & 5,9 & 3 & 3,9 & 1 & 2,5 & 0 & 0,0 & 0 & 0,0 & 2 & 8,0 & 4 & 4,0 \\
\hline $2^{\circ}$ grau inc. & 0 & 0,0 & 0 & 0,0 & 0 & 0,0 & 0 & 0,0 & 0 & 0,0 & 0 & 0 & 0 & 0,0 & 0 & 0,0 & 28 & 0,0 & 0 & 0,0 \\
\hline $2^{\circ}$ grau comp. & 2 & 2,1 & 0 & 0,0 & 10 & 5,8 & 13 & 25,5 & 6 & 7,8 & 5 & 12,6 & 0 & 0,0 & 0 & 0,0 & 0 & 5,1 & 18 & 18,0 \\
\hline Nível Superior & 0 & 0,0 & 0 & 0,0 & 2 & 1,2 & 8 & 15,7 & 1 & 1,3 & 18 & 45,1 & 0 & 0,0 & 1 & 100 & 18 & 0,8 & 27 & 27,0 \\
\hline Desconhecido & 17 & 17,9 & 5 & 62,5 & 28 & 16,0 & 26 & 50,9 & 15 & 19,4 & 15 & 37,6 & 0 & 0,0 & 0 & 0,0 & 3 & 17,0 & 46 & 46,0 \\
\hline Total & 95 & 100,0 & 8 & 100,0 & 174 & 100,0 & 51 & 100,0 & 77 & 100,0 & 40 & 100,0 & 6 & 100,0 & 1 & 100,0 & 60 & 100,0 & 100 & 100 \\
\hline
\end{tabular}

$\mathrm{HE}=$ Hospital Escola, $\mathrm{HA}=$ Hospital Privado, $1^{\circ} \mathrm{grau}$ inc. $=1^{\circ} \mathrm{grau}$ incompleto, $1^{\circ} \mathrm{grau}$ comp. $=1^{\circ} \mathrm{grau}$ completo, $2^{\circ} \mathrm{grau}$ inc. $=2^{\circ} \mathrm{grau}$

incompleto, $2^{\circ}$ grau comp. $=2^{\circ}$ grau completo

\section{Tabela 6}

Distribuição das puérperas estudadas no Hospital Escola da Faculdade de Medicina do Triângulo Mineiro, e no hospital particular, segundo o número de gestações e idade. Uberaba, Minas Gerais, Brasil, 1996.

\begin{tabular}{|c|c|c|c|c|c|c|c|c|c|c|c|c|c|c|c|c|c|c|c|c|}
\hline \multirow{4}{*}{$\begin{array}{l}\text { Número } \\
\text { de } \\
\text { Gestações }\end{array}$} & \multicolumn{20}{|c|}{ Idade } \\
\hline & \multicolumn{4}{|c|}{$<20$} & \multicolumn{4}{|c|}{$201-30$} & \multicolumn{4}{|c|}{$30 \mathrm{I}-40$} & \multicolumn{4}{|c|}{40 I- 50} & \multicolumn{4}{|c|}{ Total } \\
\hline & \multicolumn{2}{|c|}{$\mathrm{HE}$} & \multicolumn{2}{|c|}{ HA } & \multicolumn{2}{|c|}{$\mathrm{HE}$} & \multicolumn{2}{|c|}{$\mathrm{HA}$} & \multicolumn{2}{|c|}{$\mathrm{HE}$} & \multicolumn{2}{|c|}{$\mathrm{HA}$} & \multicolumn{2}{|c|}{$\mathrm{HE}$} & \multicolumn{2}{|c|}{$\mathrm{HA}$} & \multicolumn{2}{|c|}{$\mathrm{HE}$} & \multicolumn{2}{|c|}{$\mathrm{HA}$} \\
\hline & $n$ & $\%$ & $n$ & $\%$ & $n$ & $\%$ & $\mathrm{n}$ & $\%$ & $n$ & $\%$ & $\mathrm{n}$ & $\%$ & $n$ & $\%$ & $\mathrm{n}$ & $\%$ & $\mathrm{n}$ & $\%$ & $\mathrm{n}$ & $\%$ \\
\hline Uma & 76 & 80,1 & 8 & 100,0 & 51 & 29,3 & 24 & 47,0 & 10 & 13,0 & 17 & 42,5 & 1 & 16,7 & 1 & 100,0 & 138 & 39,2 & 50 & 50,0 \\
\hline Duas & 16 & 16,8 & 0 & 0,0 & 32 & 18,4 & 20 & 39,2 & 12 & 15,6 & 10 & 25,0 & 0 & 0,0 & 0 & 0,0 & 60 & 17,0 & 30 & 30,0 \\
\hline Três & 2 & 2,1 & 0 & 0,0 & 48 & 27,6 & 3 & 5,9 & 20 & 25,9 & 9 & 22,5 & 1 & 16,7 & 0 & 0,0 & 71 & 20,2 & 12 & 12,0 \\
\hline Quatro & 1 & 1,0 & 0 & 0,0 & 24 & 13,8 & 1 & 2,0 & 14 & 18,2 & 1 & 2,5 & 1 & 16,7 & 0 & 0,0 & 40 & 11,4 & 2 & 20,0 \\
\hline Cinco & 0 & 0,0 & 0 & 0,0 & 11 & 6,3 & 0 & 0,0 & 7 & 9,1 & 1 & 2,5 & 1 & 16,7 & 0 & 0,0 & 19 & 5,4 & 1 & 1,0 \\
\hline Seis & 0 & 0,0 & 0 & 0,0 & 5 & 2,9 & 0 & 0,0 & 6 & 7,8 & 0 & 0,0 & 2 & 33,2 & 0 & 0,0 & 13 & 3,7 & 0 & 0,0 \\
\hline Sete & 0 & 0,0 & 0 & 0,0 & 2 & 1,1 & 0 & 0,0 & 5 & 6,5 & 0 & 0,0 & 0 & 0,0 & 0 & 0,0 & 7 & 2,0 & 0 & 0,0 \\
\hline Mais de sete & 0 & 0,0 & 0 & 0,0 & 1 & 0,6 & 0 & 0,0 & 3 & 3,9 & 0 & 0,0 & 0 & 0,0 & 0 & 0,0 & 4 & 1,1 & 0 & 0,0 \\
\hline Desconhecido & 0 & 0,0 & 0 & 0,0 & 0 & 0,0 & 3 & 5,9 & 0 & 0,0 & 2 & 5,0 & 0 & 0,0 & 0 & 0,0 & 0 & 0,0 & 5 & 5,0 \\
\hline Total & 95 & 100,0 & 8 & 100,0 & 17 & 100,0 & 51 & 100,0 & 77 & 100,0 & 40 & 100,0 & 6 & 100,0 & 1 & 100,0 & 352 & 100,0 & 100 & 100,0 \\
\hline
\end{tabular}

$\mathrm{HE}=$ Hospital Escola, $\mathrm{HA}=$ Hospital Privado 
Distribuição dos casos de cesariana no Hospital Escola da Faculdade de Medicina do Triângulo Mineiro e no hospital particular, segundo sua indicação. Uberlândia, Minas Gerais, Brasil, 1996.

\begin{tabular}{|c|c|c|c|c|}
\hline \multirow[b]{3}{*}{ Tipo de parto } & \multicolumn{4}{|c|}{ Freqüência } \\
\hline & \multicolumn{2}{|c|}{ HE } & \multicolumn{2}{|c|}{ HA } \\
\hline & $\mathrm{n}$ & $\%$ & $\mathrm{n}$ & $\%$ \\
\hline Cesárea iterativa & 94 & 26,7 & 36 & 36,0 \\
\hline Distócia de apresentação & 79 & 22,4 & 36 & 36,0 \\
\hline Sofrimento fetal agudo & 64 & 18,2 & 2 & 2,0 \\
\hline Apresentação pélvica em primípara & 20 & 5,7 & 5 & 5,0 \\
\hline Falha de indução & 17 & 4,8 & 0 & 0,0 \\
\hline Sofrimento fetal crônico & 15 & 4,3 & 1 & 1,0 \\
\hline Eclâmpsia & 14 & 4,0 & 0 & 0,0 \\
\hline DPP & 10 & 2,8 & 1 & 1,0 \\
\hline Primípara idosa & 9 & 2,6 & 7 & 7,0 \\
\hline Prolapso de cordão & 6 & 1,7 & 0 & 0,0 \\
\hline Outras & 24 & 6,8 & 12 & 12,0 \\
\hline Total & 352 & 100,0 & 100 & 100,0 \\
\hline
\end{tabular}

$\mathrm{HE}=$ Hospital Escola, HA = Hospital Privado, DPP = Deslocamento Prematuro de Placenta

\section{Discussão}

Nossos resultados permitiram verificar um alto índice de cesariana $(89,3 \%)$ em um hospital privado contra $24,4 \%$ do Hospital Escola. A taxa de $89,3 \%$ é alta e os motivos para explicá-las são as causas médicas e socioculturais. A cesariana em seus primórdios era acompanhada de altas taxas de morbidade e mortalidade materna, principalmente por falhas de conhecimentos técnicos. 14 A partir do aperfeiçoamento das técnicas, a cesariana começou a substituir o fórceps e suas taxas foram elevando-se devido às indicações por apresentações pélvicas, à melhoria do diagnóstico de sofrimento fetal e às cesarianas iterativas. No entanto, a taxa de cesariana no HE, é próxima aos índices recomendados pela Organização Mundial de Saúde (OMS).15 A conduta acadêmica adotada, obedecendo as indicações obstétricas no HE, é a responsável pela menor taxa em comparação com o HA.

Quanto à procedência das pacientes verificouse que a grande maioria eram da zona urbana de Uberaba e somente $12 \%$ dos casos tiveram necessidade de encaminhamento, demonstrando assim que o "parto" tem uma alta resolutividade em sua própria localidade.

A indicação de cesariana nos hospitais aqui estudados demonstrou que a cesárea iterativa é a princi- pal indicação tanto no HE quanto no HA. Esse resultado é interessante e demonstra que a maior liberalidade de indicação de cesariana na clínica particular está vinculada à cesariana iterativa como principal causa. Como segunda causa de indicação têmse as distócias nos dois hospitais, sendo essa predominante no HA, ocorrendo provavelmente por uma espera inadequada da evolução do parto. O diagnóstico de sofrimento fetal é cerca de nove vezes mais freqüente no Hospital Escola, que, por ser um hospital de referência, recebe em maior proporção as patologias obstétricas.

O índice de cesariana foi menor entre as pacientes do HE, que tiveram menor escolaridade, ocorrendo o oposto entre aquelas do HA. Autores como Gentile de Melo, 16 Estelita-Lins e Janowitz, 17 relataram que a indicação de cesariana está relacionada ao melhor nível socioeconômico; já Tussing e Wojtowyez 18 ao analisarem o aumento das taxas de cesarianas, relatam que o fato está também ligado ao conforto médico e da paciente, haja vista que tais situações ocorrem comumente, embora não sejam frequentemente mencionadas.

Conclui-se que as principais indicações de cesariana nos dois hospitais são as mesmas, entretanto, a maior proporção de cesariana iterativa e distócia de apresentação ocorreram no hospital privado, provavelmente ligadas a fatores médicos. 


\section{Referências}

1. Sperling LS, Henriksen TB, Ulrichsen H, Hedegard M, Moller H, Hansen V, Ulisen B, Secher NJ. Indications for cesarean section in singleton pregnancies in two danish countles with different. Acta Obstet Gynecol Scand 1994; 73: 129-35.

2. Taffel SM. Cesarean section in América: dramatic trends, 1970 to 1987. Stat Bull Metrop Insur 1989; 70: 2-11.

3. Miranda S, Miranda GV, Correa MD. Indicações de cesariana no Hospital das Clínicas da UFMG durante duas décadas. RBGO: Rev. Bras. Ginecol Obstet 1996; 18: 511-4.

4. Osava RH. A redução das taxas de operações cesarianas no Brasil: um desafio para a enfermagem obstétrica. J Bras Ginecol 1996; 106: 421-7.

5. Souto JCS, Cardozo SE, Schwartz IVD, Passos EP. Cesariana e parto vaginal no Hospital das Clínicas de Porto Alegre em 1988. J Bras Ginecol 1993; 103: 297-301.

6. Miranda S. Evolução da incidência e indicações da operação cesariana no período de 1970 a 1987, Maternidade Otto Cirne, Hospital das Clínicas da UFMG [dissertação mestrado]. Belo Horizonte: Centro de Ciências da Saúde da Universidade Federal de Minas Gerais; 1988.

7. Betiol, H. Saúde perinatal em Ribeirão Preto: estudo de algumas variáveis sociais e biológicas no perfil reprodutivo de mães adolescentes [dissertação mestrado]. Ribeirão Preto: Faculdade de Medicina da Universidade de São Paulo; 1990.

8. Fabri RH, Gomes UA. Estudo da ocorrência dos diferentes tipos de partos em Uberaba-MG. J Bras Ginecol 1998; 108: 43-8.
9. Berkowitz GS, Flarman GS, Mojica MA, Bauman J, Reg RH. Effect of physician characteristics on the cesarean birth rate. Am J Obstet Gynecol 1989; 161: 146-9.

10. Gleicher N. Cesarean section rates in the United States. JAMA 1984; 252: 3273-6.

11. Maia Filho NL, Mathias L, Iliar J, Pientznauer R, Carvalho PM. Operação cesariana: a propósito de suas indicações. J Bras Ginecol 1994; 104: 423-6.

12. Muylder X, Thiery M. The cesarean delivery rate can be safely reduced in a developing country. Obstet Gynecol 1990; 75: 360-4.

13. Turner MJ, Brassil M, Gordon H. Active management of labor associated with a decrease in the cesarean section rate in nullipars. Obstet Gynecol 1988; 71: 150-4.

14. Murta EFC, Gonçalves RC, Carvalho LM, Veludo KP, Ribeiro JU, Salum R. Histerectomia no ciclo gravídicopuerperal: estudo retrospectivo de 15 anos. J Bras Ginecol 1995; 105: 361-7.

15. Ministério da Saúde. Assistência integral à saúde da mulher: bases de ação programática. Brasília: Centro Documentação do Ministério da Saúde; 1984.

16. Gentile de Melo C. Privatização e produtividade do serviços de saúde: pesquisa setorial sobre a incidência de cesárea. Rev Paul Hosp 1969; 17: 3-11.

17. Estellita-Lins F, Janowitz B. Operação cesariana no Sudeste do Brasil. Femina 1982; 10: 91-103.

18. Tussing AD, Wojtowyez MA. The cesarean decision in New York State 1996: economic and noneconomic aspects. Med Care 1992; 30: 529-40. 